\title{
EpCAM Expression Is an Indicator of Increased Incidence of Relapse in p53-Positive Breast Cancer
}

\author{
Federico Ambrogi ${ }^{1}$, Marco Fornili ${ }^{1}$, Saverio Alberti ${ }^{2}$, Patrizia Querzoli ${ }^{3}$, Mauro Piantelli ${ }^{2}$, Massimo Pedriali ${ }^{3}$, \\ Patrizia Boracchi ${ }^{1}$, Elia Biganzoli ${ }^{1,4} \&$ Danila Coradini ${ }^{1}$ \\ ${ }^{1}$ Department of Clinical and Community Health Sciences, Medical Statistics, Biometry and Bioinformatics, \\ University of Milan, Milan, Italy \\ ${ }^{2}$ Unit of Cancer Pathology, Department of Oncology and Neurosciences and CeSI, Fondazione 'G. D'Annunzio', \\ University of Chieti, Chieti, Italy \\ ${ }^{3}$ Section of Surgical Pathology, Department of Experimental and Diagnostic Medicine, University of Ferrara, \\ Ferrara, Italy \\ ${ }^{4}$ Fondazione IRCCS, Istituto Nazionale Tumori, Milan, Italy \\ Correspondence: Danila Coradini, Department of Clinical and Community Health Sciences, Medical Statistics, \\ Biometry and Bioinformatics, University of Milan, Via Vanzetti 5, 20133 Milan, Italy. E-mail: \\ danila.coradini@yahoo.it
}

Received: September 25, 2012 Accepted: October 11, 2012 Online Published: December 19, 2012

doi:10.5539/cco.v2n1p40 URL: http://dx.doi.org/10.5539/cco.v2n1p40

\begin{abstract}
Originally identified as Trop1, epithelial cell adhesion molecule (EpCAM) is a transmembrane glycoprotein that received great attention because of its putative involvement in metastatic spread of several solid tumors including breast cancer. Experimental evidence indicated that EpCAM is a key transcriptional target of p53 tumor suppressor, due to the presence of specific p53 response elements within EPCAM gene promoter. Aim of present study was to investigate the joined prognostic significance of p53 and EpCAM in a cases series of 640 breast cancers with long-term follow-up. In addition, considering the role of EpCAM in modulating cell-cell interaction by decreasing the cytoskeleton-anchored fraction of E-cadherin, when feasible, we evaluated also E-cadherin expression. Results indicated that EpCAM overexpression was associated with a high incidence of relapse and that, when in association with p53 status, EpCAM was able to identify, within p53-positive cases, those with the highest incidence of relapse. Conversely, E-cadherin overexpression was associated with a low incidence of relapse. Overall, these findings are of particular clinical relevance taking into account the biological link between p53 activity and EPCAM gene expression and the functional relationship between EpCAM and E-cadherin in mediating cell-to-cell adhesion.
\end{abstract}

Keywords: EpCAM expression, p53 status, breast cancer, prognosis

\section{Introduction}

Since its discovery in 1979, an intensive investigation has clearly proved that p53 is a pivotal tumor suppressor playing a central role in cell growth control by induction of apoptosis, cell-cycle arrest and senescence in response to a plethora of cellular stress signals (Levine \& Oren, 2009; Suzuki, 2011). TP53 gene is mutated in more than half of human cancers, and mutation frequently results in the expression/accumulation of an inactive protein, which hampers the correct cell cycle control and apoptosis activation (Olivier, 2010). Clinical evidence indicated that both gene mutations and protein accumulation are associated with poor prognosis (Cheok, 2011).

As regards breast cancer, p53 is mutated in about $30-40 \%$ of cases (Olivier, 2006), with a high frequency in some tumor subtypes including the pathologic class of triple-negative breast cancers (TNBCs). These tumors are characterized by a negative estrogen (ER) and progesterone receptor (PR) status, a negative HER2 expression, and the immunohistochemical expression of basal cytokeratin and epidermal growth factor receptor (EGFR) (Di Leo, 2007; Langerod, 2007; Chae, 2009). Recently, we found that p53 protein expression is able to subdivide this so critical pathologic class of breast tumors into two distinct subsets with a different outcome, being p53-expressing tumors associated with short overall and event-free survival (Biganzoli, 2011).

Originally called Trop-1 and also known as ESA and GA733-2, epithelial cell adhesion molecule (EpCAM) is a 
transmembrane glycoprotein that received great attention because of its putative involvement in metastatic spread of several solid tumors including breast cancer (Cimino, 2010; Spizzo, 2011). Initially identified as a carcinoma-associated antigen (Schön, 1993), EpCAM was found expressed also on most normal epithelial cells where it functions as a homotypic calcium-independent cell adhesion molecule between epithelial cells and intraepithelial lymphocytes (Litvinov, 1994). In normal epithelial tissues, including the luminal epithelium of mammary gland (Momburg, 1987), EpCAM localizes to the basolateral membrane whereas in carcinomas (including breast cancer) its expression pattern shifts to an intense membranous overexpression. EpCAM membranous overexpression has provided clinical evidence as prognosticator in several human carcinomas (Went, 2004; Went, 2008; Kimura, 2007; Seligson, 2004) including breast cancer, where it correlated with poor disease-free and overall survival in node-negative as well as in node-positive patients (Gastl, 2000; Schmidt, 2008; Spizzo, 2004).

Despite EpCAM expression has been associated with tumor aggressiveness, its real biological function remains unclear. On one hand, EpCAM physiological functions include cellular differentiation and maturation during embryonic development and initiation of tissue regeneration after inflammatory response (Trzpis, 2007). On the other hand, it can affect cell cycle control upregulating the proto-oncogene c-Myc (Munz, 2004) and disrupts cell adhesion by decreasing the cytoskeleton-anchored fraction of E-cadherin (Litvinov, 1997).

Experimental evidence indicated that EpCAM is a key transcriptional target of p53 because the presence of specific response elements within EPCAM gene promoter (Sankpal, 2009).

Based on the relationship between p53 and EpCAM expression, we investigated their joined prognostic significance in a large cases series of breast cancers with long-term follow-up. In addition, considering the role of EpCAM in modulating cell-cell interaction by decreasing the cytoskeleton-anchored fraction of E-cadherin, when feasible we evaluated also the joined effect of E-cadherin expression.

\section{Method}

\subsection{Patients Cases Series}

Seven-hundred consecutive patients from the Ferrara Cancer Register treated for a primary breast cancer between January 1989 and December 1993 at the Surgical Units of Ferrara S. Anna Hospital-University or at Surgical Units of the Ferrara province's hospitals were retrospectively included in this study. Informed written consent was obtained from all patients and the University of Ferrara Research Ethics Committee approved the study. Eligible criteria were pathologic stage $\mathrm{T} 1$ to T3, availability of at least 10 resected axillary lymph nodes, absence of synchronous bilateral tumors or any other malignancy before breast cancer diagnosis and up to 6 months after surgery, absence of distant metastases at diagnosis and up to 6 months after surgery, and no neo-adjuvant therapy. At diagnosis, 392 patients were classified as node-negative (pN-) and 308 as node-positive $(\mathrm{pN}+)$.

According to treatment protocols applied, 335 of them received an adjuvant therapy. Clinical baseline and patient's follow-up data (date and site of relapse, last follow-up time, and date and cause of death) were extracted from the Ferrara Cancer Registry. Data on patient age, tumor histology, pathological stage (pT), grading, and number of involved axillary lymph nodes were also collected (Ambrogi, 2006). After immunohistochemical assessment of routine biomarkers (ER, PR, HER2 and p53) for 640 patients (Table 1), a residual paraffin-embedded tissue material of the primary tumor was available for the immunohistochemical evaluation of EpCAM and E-cadherin expression.

The recommendations for Tumor Marker Prognostic Studies (REMARK) criteria were followed throughout, as recommended by McShane et al. (2005). The protocol of this study was approved by the board of the Ministry of the University and Research ("Identification and validation of new markers of metastasizing phenotype of breast cancer”, prot. MM06095812_006, year 2000).

\subsection{Immunohistochemistry (IHC)}

Breast cancer tissue microarrays (TMA) were prepared as previously described (Alberti, 2012). Then, the sections were processed in a microwave oven in a TEC buffer (Tris-citrate-EDTA), $\mathrm{pH} 7.8$, to unmask antigenic sites after formalin fixation. IHC was performed with an automated immunostainer (Ventana NEXES, Medical System, Tucson, AZ, USA). Slides were stained for EpCAM using the VU-ID9 antibody (NovoCastra Laboratories Ltd., Newcastle upon Tyne, UK) or for E-cadherin using the ECH-6 antibody (Medite, Castelnuovo Del Garda, Italy). Vectastain ABC peroxidase kit (Vector Laboratories, DBA Italia, Segrate, Italy) was used to reveal antibody binding. Slides treated with normal serum or isotype-matched antibody, were used as negative controls. Endogenous biotin was saturated with a biotin blocking kit (Vector Laboratories). Two pathologists 
independently examined all TMA sections. For each tumor, at least 400 cells were counted and the percentage of immunostained cells was recorded. In both cases, protein expression was scored according to the product between the intensity coefficient ( 0 , negative; 1 , low; 2,moderate; 3 , strong) and the frequency of positive coefficient $(0$, no colored cells; $1,1-9 \% ; 2,10-49 \% ; 3,50-79 \% ; 4,80-100 \%)$, then categorized as follows: 0 , negative score; 1+, score 1-4; 2+, score 5-8; 3+, score 9-12. All routinely evaluated biological variables were categorized according to conventional cut-offs (Table 1). P53 status was considered as positive (p53+) when a percentage greater than 10 of positive staining cells was observed.

According to Spizzo et al. (2004), who defined a total score $>4$ as EpCAM overexpression, in the statistical analysis, we dichotomized EpCAM expression in low-to-nil (i.e., categories 0 and $1+$, corresponding to a total score $\leq 4$ ) and intermediate/high (i.e., categories $2+$ and $3+$, corresponding to a total score $>4$ ).

\subsection{Statistical Analysis}

To evaluate the association between EpCAM and other patho-biological variables adjusted odds ratios were estimated by multiple logistic regressions. The multivariate associations among EpCAM, p53 and other patho-biological variables were also investigated and visualized through multiple correspondence analysis (MCA) that visualizes on a bi-dimensional plot the association of both categorical and continuous variables (Greenacre, 2007). MCA has the advantage of implying neither linearity nor specific distribution characteristics and to visualize association patterns between markers. The use of a bidimensional plot, easy to interpret, is possible at the expense of losing some information on the pattern of associations. The markers are represented in a space defined by factorial axes, characterized by a percentage if inertia (i.e., quantifying the information explained by each axis). The distance between marker categories (defined according to a $\chi^{2}$. metric) indicates their dissimilarity.

The effect of EpCAM and p53 on patient's outcome was evaluated considering as endpoints the occurrence of any first relapse (recurrence, distant metastases, contralateral tumor, other neoplasias whichever occurred first) over the follow-up period and death without evidence of disease as competing risk. Accordingly, in the analysis, methodologies accounting for the presence of competing risks were used. Nonparametric estimates of the probability of occurrence of first adverse events [crude cumulative incidence (CCI)] were obtained (Marubini, 1995). A procedure based on the proportional subdistribution hazard regression model was used to assess the difference among CCI curves, both in univariate and multivariate analysis, according to the different expression levels of EpCAM and p53 (Fine, 1999). This model is equivalent of Cox regression model in the presence of competing risks. In multivariate analysis the effects of EpCAM and p53 were adjusted for established prognostic factors; in particular, pathologic T stage (T2-T3 versus T1), grading (G2 and G3 versus G1), age, ER protein level (in log-scale), HER2, E-cadherin and number of metastatic lymph nodes (1-3, 4-9 and >9 versus 0 ) were considered. The null hypothesis of regression coefficients equal to 0 was tested by Wald test.

The median follow-up was estimated by reversed Kaplan-Meier method (Schemper, 1996). R software (R Foundation, 2011) was utilized throughout this study.

\section{Results}

Table 1 summarized the associations between EpCAM expression and patho-biological variables. There was an evidence of association with tumor size $(\mathrm{P}=0.03)$, ER $(\mathrm{P}=0.002)$ and $\mathrm{E}$-cadherin expression $(\mathrm{P}<0.00001)$. In particular, with respect to pT1 tumors, which were prevalently EpCAM-negative (score $<4)(340 / 411,82.7 \%$ ), $38.5 \%$ of large tumors (pT3) were EpCAM-positive. Similarly, with respect to ER-positive tumors, which were prevalently EpCAM-negative $(364 / 419,86.9 \%), 32 \%$ of ER-negative specimen were EpCAM-positive. The finding supports the clinical evidence that high EpCAM expression is generally found in tumors potentially more aggressive. As regards the association between EpCAM and E-cadherin, we found a positive association and an overall agreement of 73.7\%: 383/593 tumors showed a low-to-nil expression of both adhesion molecules whereas 54/593 overexpressed both proteins. No significant association was found between EpCAM expression and tumor histotype or grade, lymph node status, PR, HER2 or p53 expression. No statistically significant association was observed between EpCAM expression and patient age. However, with respect to young or elderly women, patients with age ranging from 51 to 55 years (the interval of age in which epidemiologically peaks the incidence of breast cancer) showed an increased percentage of EpCAM-positive tumors. In fact, while only $19.6 \%$ young and $15.1 \%$, elderly women had an EpCAM-positive tumor, patients with age ranging from 51 to 55 years had an EpCAM-positive tumor in a $30.3 \%$ of cases $(\mathrm{OR}=1.99, \mathrm{IC}=0.50-7.90)$ maybe as a consequence of the cellular stress induced by unbalanced hormonal stimulus associated with perimenopausal status. 
Table 1. EpCAM expression and patho-biological features $(n=640)$

\begin{tabular}{|c|c|c|c|c|}
\hline Categorical variables & $\begin{array}{l}\text { Overall } \\
\mathrm{N}(\%)\end{array}$ & $\begin{array}{l}\text { Negative (score } \\
\leq 4) \mathrm{N}(\%)\end{array}$ & $\begin{array}{l}\text { Positive } \\
(\text { score }>4) \mathrm{N}(\%)\end{array}$ & $O R$ (P value) \\
\hline \multicolumn{5}{|l|}{ Age $\left(n=640 ; \chi^{2}=6.37, P=0.17\right)$} \\
\hline $34-40$ & $46(7.2)$ & $37(7.1)$ & $9(7.8)$ & 1 \\
\hline $41-50$ & $134(20.9)$ & $116(22.1)$ & $18(15.7)$ & $0.60(0.42)$ \\
\hline $51-55$ & $76(11.9)$ & $53(10.1)$ & $23(20.0)$ & $1.99(0.33)$ \\
\hline $56-70$ & $252(39.4)$ & $207(39.4)$ & $45(39.1)$ & $1.01(0.99)$ \\
\hline $71-90$ & $132(20.6)$ & $112(21.3)$ & $20(17.4)$ & $0.57(0.44)$ \\
\hline \multicolumn{5}{|c|}{ Histotype $\left(n=640 ; \chi^{2}=2.69, P=0.26\right)$} \\
\hline Ductal & $481(75.2)$ & $384(73.1)$ & $97(84.3)$ & 1 \\
\hline Lobular & $100(15.6)$ & $93(17.7)$ & $7(6.1)$ & $0.37(0.13)$ \\
\hline Other types & $59(9.2)$ & $48(9.2)$ & $11(9.6)$ & $1.37(0.61)$ \\
\hline \multicolumn{5}{|c|}{ Tumor size $\left(n=638 ; \chi^{2}=7.18, P=\mathbf{0 . 0 3}\right)$} \\
\hline $\mathrm{pT} 1$ & $411(64.4)$ & $340(65.0)$ & $71(61.7)$ & 1 \\
\hline pT2 & $214(33.6)$ & $175(33.5)$ & $39(33.9)$ & $1.23(0.56)$ \\
\hline pT3 & $13(2.0)$ & $8(1.5)$ & $5(4.4)$ & $16.18(\mathbf{0 . 0 1})$ \\
\hline \multicolumn{5}{|c|}{ Grading $\left(n=639 ; \chi^{2}=2.64, P=0.27\right)$} \\
\hline G1 & $121(18.9)$ & $103(19.6)$ & $18(15.8)$ & 1 \\
\hline $\mathrm{G} 2$ & $388(60.7)$ & $320(61.0)$ & $68(59.6)$ & $1.52(0.37)$ \\
\hline $\mathrm{G} 3$ & $130(20.4)$ & $102(19.4)$ & $28(24.6)$ & $2.38(0.11)$ \\
\hline \multicolumn{5}{|l|}{ Nodal status $(n=640)$} \\
\hline Negative & $357(55.8)$ & $298(56.8)$ & $59(51.3)$ & 1 \\
\hline Positive & $283(44.2)$ & $227(43.2)$ & $56(48.7)$ & $1.05(0.90)$ \\
\hline \multicolumn{5}{|l|}{ Estrogen receptor $(n=532)$} \\
\hline$\leq 10 \%$ & $113(21.2)$ & $77(17.5)$ & $36(39.6)$ & 1 \\
\hline$>10 \%$ & $419(78.8)$ & $364(82.5)$ & $55(60.4)$ & $0.23(\mathbf{0 . 0 0 2})$ \\
\hline \multicolumn{5}{|l|}{ Progesterone receptor $(n=527)$} \\
\hline$\leq 10 \%$ & $159(30.2)$ & $120(27.3)$ & $39(44.3)$ & 1 \\
\hline$>10 \%$ & $368(69.8)$ & $319(72.7)$ & $49(55.7)$ & $1.03(0.95)$ \\
\hline \multicolumn{5}{|l|}{ HER $2 /$ neu $(n=630)$} \\
\hline$\leq 10 \%$ & $433(68.7)$ & $356(69.1)$ & $77(67.0)$ & 1 \\
\hline$>10 \%$ & $197(31.3)$ & $159(30.9)$ & $38(33.0)$ & $0.89(0.73)$ \\
\hline \multicolumn{5}{|l|}{$p 53(n=620)$} \\
\hline$\leq 10 \%$ & $283(45.6)$ & $224(44.0)$ & $59(53.2)$ & 1 \\
\hline$>10 \%$ & $337(54.4)$ & $285(56.0)$ & $52(46.8)$ & $0.72(0.30)$ \\
\hline \multicolumn{5}{|l|}{ E-cadherin score $(n=593)$} \\
\hline $0 / 1+$ & $437(73.7)$ & $383(79.0)$ & $54(50.0)$ & 1 \\
\hline $2+/ 3+$ & $156(26.3)$ & $102(21.0)$ & $54(50.0)$ & $\begin{array}{l}7.36 \\
(<\mathbf{0 . 0 0 0 0 1 )}\end{array}$ \\
\hline \multicolumn{5}{|c|}{ Adjuvant therapies $\left(n=517 ; \chi^{2}=1.73, P=0.63\right)$} \\
\hline No therapy & $214(41.4)$ & $177(41.6)$ & $37(40.7)$ & 1 \\
\hline Chemotherapy (CT) & $89(17.2)$ & $73(17.1)$ & $16(17.6)$ & $0.55(0.30)$ \\
\hline Hormone therapy (HT) & $187(36.2)$ & $155(36.4)$ & $32(35.1)$ & $0.61(0.27)$ \\
\hline CT plus HT & $27(5.2)$ & $21(4.9)$ & $6(6.6)$ & $0.74(0.71)$ \\
\hline
\end{tabular}

Abbreviation: $\mathrm{pT}$ stage $=$ pathological stage; $\mathrm{OR}=$ odds ratio

Bold values are statistically significant. $\chi^{2}$ is the value of the Wald statistics obtained from the multiple logistic model and it is indicated only for variables with more than two categories 
The associations between EpCAM and other patho-biological variables, namely patient age, histologic type, tumor size, grading, axillary nodal status, ER, PR, HER2, p53 and E-cadherin were explored also by multiple correspondence analysis (MCA). As shown in Figure 1, MCA indicated that the two first axes explain the 65.7\% of total variability (respectively, $54.9 \%$ the first axis and $10.8 \%$ the second axis). Specifically, the first axis separates $E R \leq 10, P R \leq 10$, HER2 $>10, G 3$, patient age $\leq 40$ years and node-positive tumors (on the left) from $\mathrm{ER}>10, \mathrm{PR}>10, \mathrm{HER} 2 \leq 10, \mathrm{G} 1$, patient age $>56$ years and node-negative tumors (on the right). The second axis mostly separates high EpCAM and E-cadherin scores, tumor histotypes different from invasive ductal or lobular carcinoma, $\mathrm{p} 53 \leq 10$ and small tumor in size ( $\mathrm{pT}=1)$ from low EpCAM and E-cadherin scores, lobular histotype (ILC), p53>10 and large tumor ( $\mathrm{pT}>1$ ). MCA confirms the positive association between EpCAM and E-cadherin expression.

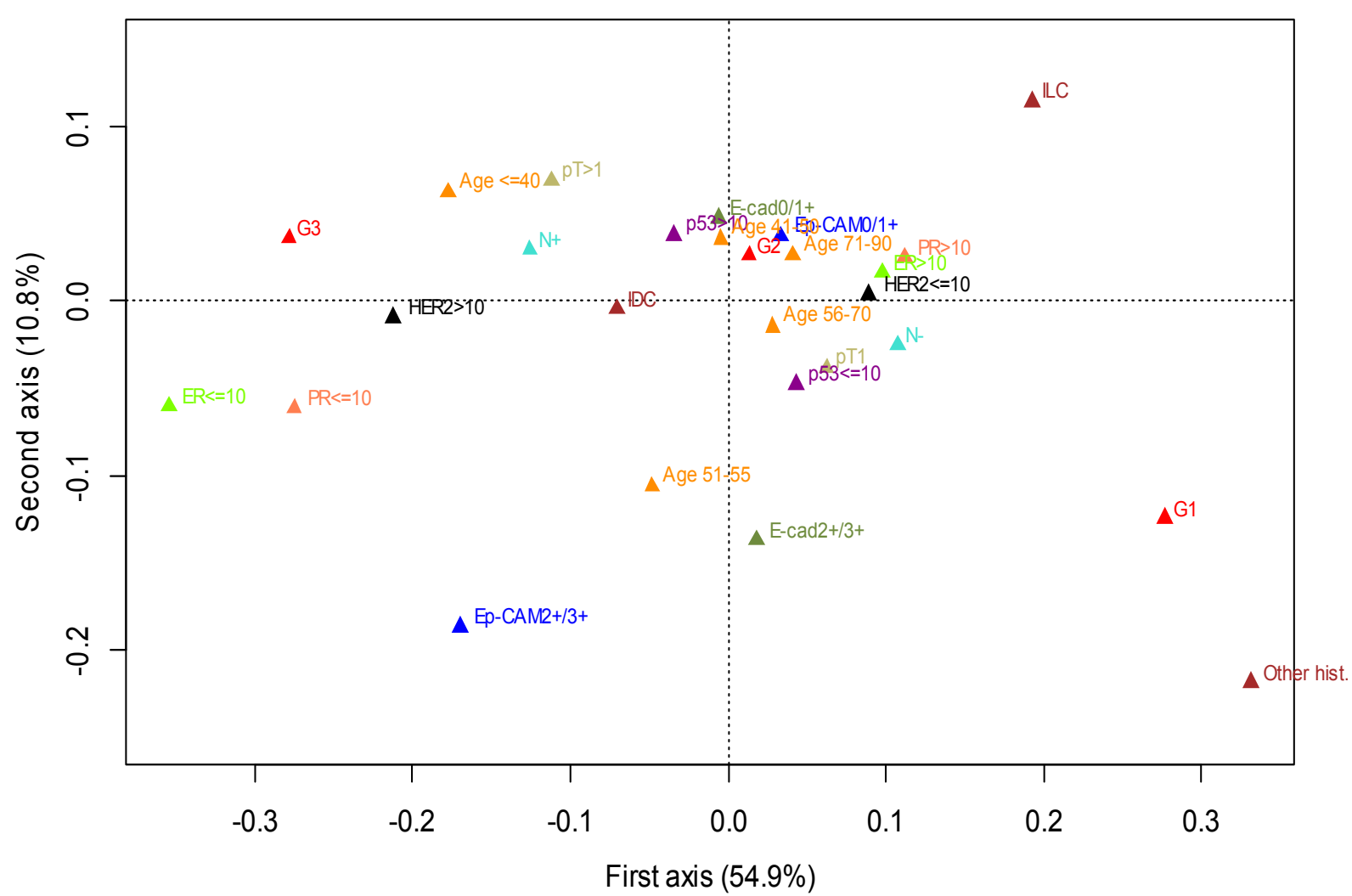

Figure 1. Associations between EpCAM expression and other clinico-biological variables. The associations were evaluated by multiple correspondence analysis. The triangles represent the categories. The distance between the labels, based on a $\chi^{2}$ metric, is a measure of the dissimilarity of the corresponding categories

The median follow-up time of the 640 patients was 99 months (range 1-157 months). Time was curtailed at 8 years when the probability of patients of being in follow-up was about $56 \%$. During this period, 96 patients developed distant metastases $(\mathrm{CCI}=15.3 \%), 48$ a local relapse $(\mathrm{CCI}=7.7 \%), 13$ a contralateral tumor $(\mathrm{CCI}=2.1 \%)$, 30 another malignancy $(\mathrm{CCI}=4.8 \%)$ and 91 dead $(\mathrm{CCI}=14.7 \%)$ as first event.

Crude cumulative incidence curves (Figure 2) showed that, as expected, patients with a tumor expressing low levels of EpCAM had a lower incidence of relapse with respect to those with an EpCAM over expressing tumor $\left(\mathrm{HR}=1.14, \mathrm{CI}=0.80-1.64, \chi^{2}=0.51, \mathrm{P}=0.47\right)$. Similarly, patients with a $\mathrm{p} 53$-negative tumor had a lower incidence of relapse with respect to patients with a 53 -positive tumor $\left(\mathrm{HR}=1.10, \mathrm{CI}=0.82-1.47, \chi^{2}=0.40, \mathrm{P}=0.53\right)$. When we analyzed the joined effect of EpCAM expression and p53 status, we found that the subgroups EpCAM-negative/p53-positive, EpCAM-positive/p53-negative and EpCAM-negative/p53-negative had similar incidence of relapse, while the subgroup EpCAM-positive/p53-positive had a highest incidence of relapse. Notably, EpCAM expression was able to discriminate among p53-positive tumors those associated with an increased incidence of relapse. 

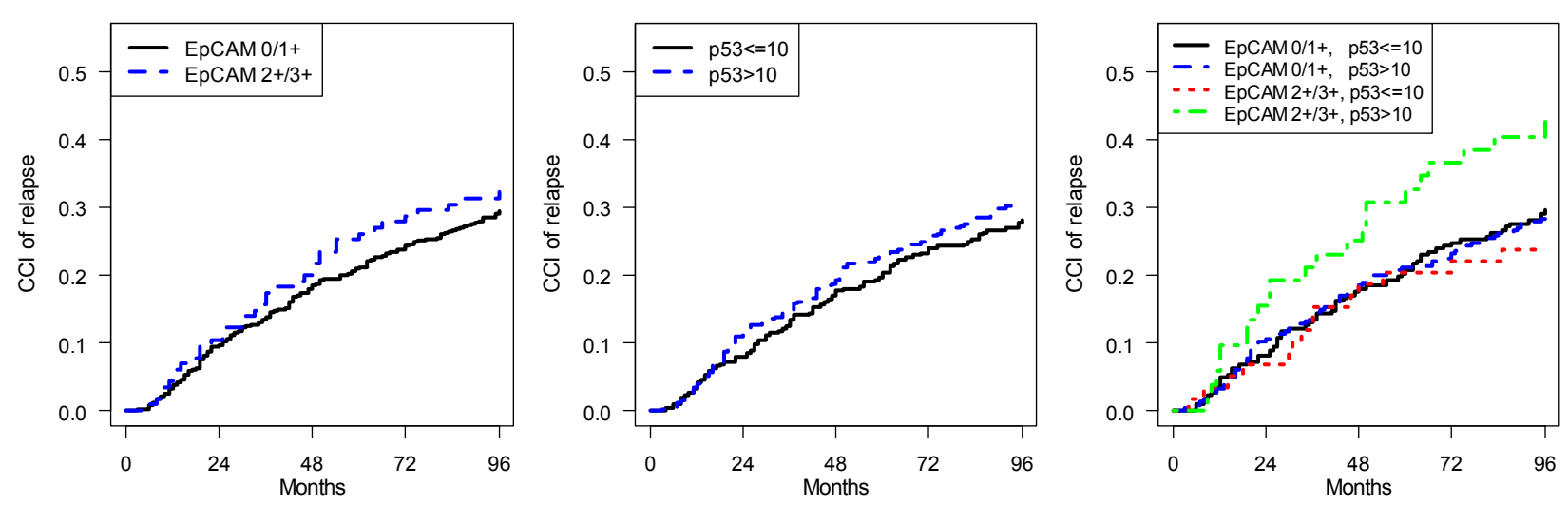

Figure 2. Crude cumulative incidences curves for EpCAM and p53 singly or in association

Analyzing the joined effect of EpCAM expression and p53 status on patients' outcome, adjusted for other prognostic factors, we found a statistically significant interaction $\left(\chi^{2}=7.50, \mathrm{P}=0.006\right)$. Conversely, no interaction was found between EpCAM and E-cadherin expression. To simplify the presentation of the regression model results, in Table 2 we included only the effect of EpCAM overexpression in p53-negative and p53-positive cases.

Table 2. Regression model EpCAM/p53

\begin{tabular}{|c|c|c|c|c|}
\hline Variable & Coefficient estimate & HR & $95 \% \mathrm{CI}$ & $P$-value \\
\hline EpCAM+ vs. EpCAM- in p53- & -0.53 & 0.59 & $0.28-1.22$ & 0.15 \\
\hline EpCAM+ vs. EpCAM- in p53+ & 0.75 & 2.11 & $1.21-3.67$ & 0.008 \\
\hline Age & -0.01 & 0.99 & $0.98-1.01$ & 0.33 \\
\hline G2 vs. G1 & -0.03 & 0.98 & $0.61-1.56$ & 0.92 \\
\hline G3 vs. G1 & -0.10 & 0.90 & $0.49-1.64$ & 0.73 \\
\hline $\mathrm{pT}>1$ vs. $\mathrm{pT}=1$ & 0.65 & 1.92 & $1.35-2.74$ & 0.0003 \\
\hline $\log (1+\mathrm{ER})$ & -0.06 & 0.94 & $0.83-1.06$ & 0.29 \\
\hline 1-3 nodes vs. 0 nodes & 0.43 & 1.53 & $1.04-2.27$ & $\mathbf{0 . 0 3}$ \\
\hline 4-9 nodes vs. 0 nodes & 0.30 & 1.36 & $0.77-2.38$ & 0.29 \\
\hline$>9$ nodes vs. 0 nodes & 0.98 & 2.67 & $1.54-4.61$ & 0.0005 \\
\hline E-cadherin $2+/ 3+$ vs. E-cadherin $0 / 1+$ & -0.19 & 0.82 & $0.55-1.24$ & 0.33 \\
\hline HER $2>10$ vs. HER $2<10$ & -0.24 & 0.79 & $0.53-1.16$ & 0.22 \\
\hline
\end{tabular}

Abbreviations: $\mathrm{CI}=$ confidence interval; $\mathrm{ER}=$ estrogen receptor; $\mathrm{HR}=$ subdistribution hazard ratio

As regards E-cadherin expression singly (Figure 3), crude cumulative incidence curves indicated that E-cadherin-negative tumors were associated with a high incidence of relapse with respect to E-cadherin-positive tumors $\left(\mathrm{HR}=0.79, \mathrm{CI}=0.56-1.12, \chi^{2}=1.77, \mathrm{P}=0.18\right)$. When we analyzed the joined effect of EpCAM and E-cadherin, we found that the subgroups EpCAM-negative/E-cadherin-negative, EpCAM-positive/E-cadherinnegative and EpCAM-positive/E-cadherin-positive had similar incidences of relapse, while the subgroup EpCAM-negative/E-cadherin-positive was associated with the lowest incidence of relapse. 

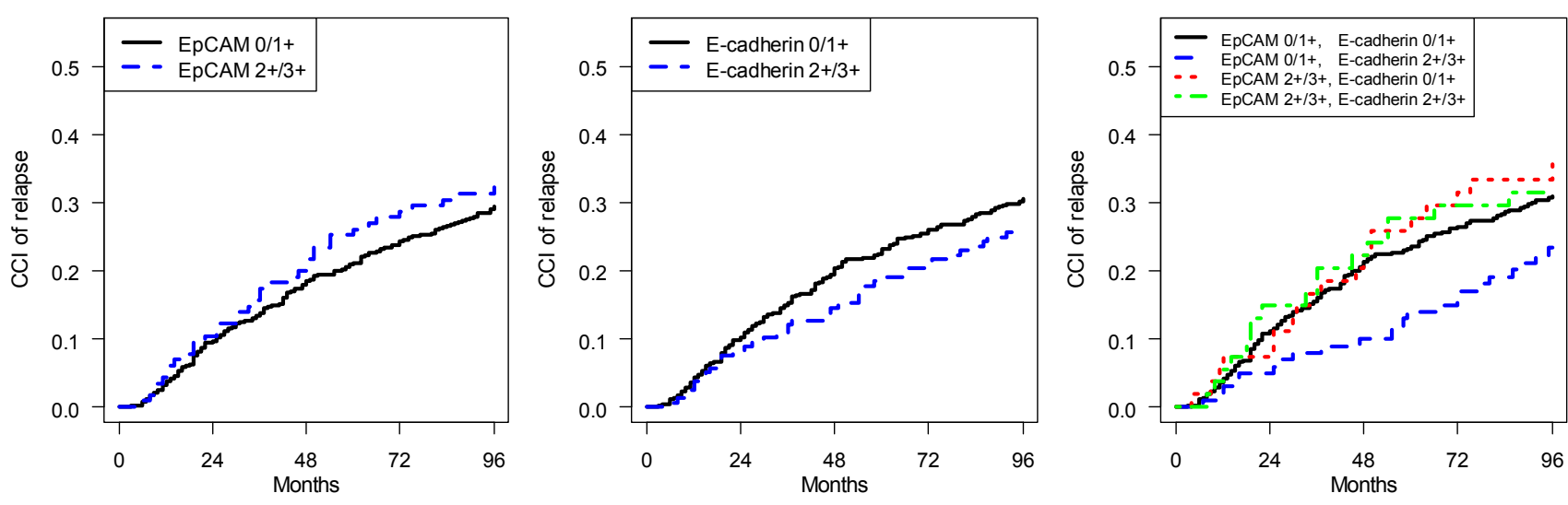

Figure 3. Crude cumulative incidences curves for EpCAM and E-cadherin singly or in association

\section{Discussion}

In response to cellular stress and DNA damage, p53 tumor suppressor blocks cell cycle progression and induces apoptosis or senescence through a wide and complex signaling pathway. Because of such a complex regulatory network, it is not surprising that an impaired p53 activity, due to TP53 gene mutation or protein inactivation, may affect many fundamental biological processes, trigger neoplastic transformation and eventually promote cancer development (Levine, 2009; Olivier, 2010). Among the plethora of genes under p53 control, there is EPCAM, which encodes for a transmembrane adhesion molecule intensively investigated because of its putative involvement in the metastatic spread of several cancers including breast cancer (Cimino, 2010; Spizzo, 2011). Experimental evidence demonstrated that in order to repress EPCAM gene transcription, p53 recognizes and binds to a specific response element within EPCAM gene promoter region (Sankpal, 2009).

In human cancers, most TP53 mutations occur within the central DNA binding domain, thus preventing the correct binding of mutant $\mathrm{p} 53$ to target gene. In the case of EPCAM gene, mutant p53 protein is unable to repress gene transcription with the subsequent constitutive expression of the protein (Figure 4). Experimental studies indicated that EpCAM may be overexpressed 100- to 1000-fold in primary and metastatic breast cancer (Osta, 2004) thus providing cancer cells for a gain of function that actually results in the acquisition of advantageous features. In fact, as a cell signaling adhesion molecule, EpCAM overexpression can promote cell proliferation, apoptosis resistance and can disrupt the functions normally exerted by other cell adhesion molecules, especially cadherins, which play an important role in defining cell fate and maintaining tissue integrity. In epithelia, this critical role belongs to E-cadherin, which is crucial for the establishment and maintenance of epithelial cell polarity, morphogenesis of epithelial tissues, and regulation of cell proliferation and programmed cell death (Takeichi, 1995; Hermiston, 1995). Indeed, E-cadherin interacts with the cytoskeleton through $\alpha$ - and $\beta$-catenins thus linking extracellular signaling to intracellular environment (Rosales, 1995). As an adhesion molecule, EpCAM should provide additional intercellular connections to cells, but it has proven to affect negatively the anchorage of E-cadherin to the cytoskeleton, thereby weakening the strength of cell-cell interactions and altering signal transduction (Winter, 2003; Litviniv, 1997).

In agreement with these findings, a very recent study (Guerra, 2012) has provided evidence that EpCAM knockout mice develop congenital tufting enteropathy, a hereditary disease characterized by enteric mucosa degeneration, through the dysregulation of intestinal E-cadherin/ $\beta$-catenin axis. These in vivo results support the functional connection between EpCAM expression and E-cadherin/ $\beta$-catenin complex previously observed in in vitro models. In addition, experimental studies, performed in breast cancer cell lines constitutively expressing high EpCAM protein levels, have clearly demonstrated that EpCAM gene silencing resulted in an improved anchorage of the E-cadherin/ $\alpha$-catenin/ $\beta$-catenin complex to the cytoskeleton associated with a dramatic inhibition of cell proliferation, migration and invasion (Osta, 2004). 


\section{a}

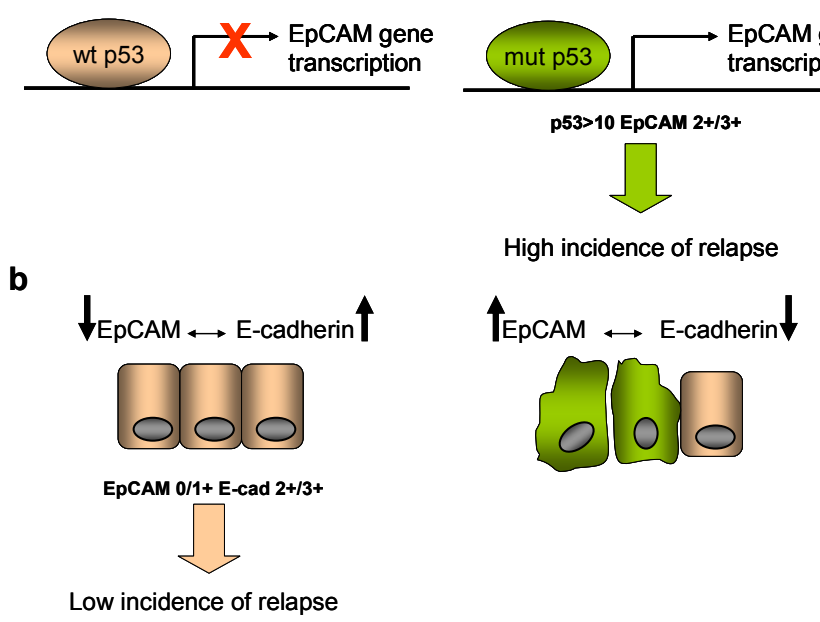

Figure 4. (a) Specific p53 response elements have been identified within EPCAM gene and functional studies in breast cancer cells have demonstrated that wild-type p53 negatively regulates EpCAM expression. Conversely, p53 loss-of-function, because of mutation, results in a significant increase in EpCAM expression. In our cases series, p53-positive/EpCAM-positive tumors were associated with a high incidence of relapse. (b) Experimental evidence indicated that EpCAM and E-cadherin expression are inversely associated and that increasing expression of EpCAM leads to the gradual abrogation of adherens junctions by decreasing the cytoskeleton -anchored fraction of E-cadherin. In our cases series, EpCAM-negative/E-cadherin-positive tumors were associated with a low incidence of relapse

Present results indicate that, as expected (Gastl, 2000; Schmidt, 2008; Spizzo, 2004), EpCAM overexpression is associated with a high incidence of relapse and that, conversely, E-cadherin overexpression is associated with a low incidence of relapse. In addition, present results indicate that when in association with p53 status, EpCAM expression is able to identify, within p53-positive tumors, those associated with the highest incidence of relapse. This finding is of particular clinical relevance taking into account the above-described biological link between p53 activity and EPCAM gene expression. In fact, although the immunohistochemical evaluation, performed on surgical specimens, provides knowledge about the mere presence of the p53 protein, nothing tells us about its functionality. Therefore, EpCAM evaluation gives us additional elements to infer, even if indirectly, on p53 protein functionality and on the putative genetic alteration liable for such a dysfunction. According to the type of mutation (point mutation, deletion, insertion or stop codon), p53 synthesis may be totally inhibited or generate functionally altered molecules. Immunohistochemically p53-positive tumors are mainly due to a protein accumulation resulting from missense mutations that allow protein synthesis but affect its function and degradation. It is well known that most missense mutations involve DNA binding domain, hence hampering the correct p53 binding to the corresponding response element on the promoter region of the target gene. According to this notion, we can assume that, within the subgroup of p53-positive tumors, those over expressing EpCAM and characterized by the highest incidence of relapse, are de facto tumors carrying a missense mutation in the DNA binding domain of TP53 gene; that is, a mutation that prevents the correct binding to EPCAM gene and subsequent transcription repression. Such a fail results in a constitutive overexpression of EpCAM protein, which provides tumor cells for a more aggressive phenotype as indicated by the fact that about half of them were hormone steroid receptors-negative and 36.5\% were HER2-positive. Conversely, as regards p53-positive tumors that do not express EpCAM and show a cumulative incidence of relapse similar to that of p53-negative tumors, a different kind of missense mutation involving ligand binding domain or protein degradation can be hypothesized. In this case, despite the protein dysfunction, EPCAM gene expression is inhibited and tumor aggressiveness is reduced as indicated by the high frequency of ER-positive (82\%) and PR-positive (76\%) cases. Such a tendency of EpCAM-positive tumors to be more aggressive is corroborated by the finding that when we considered the joined effect of EpCAM and E-cadherin expression on patients' outcome, the subgroup of patients with the lowest cumulative incidence of relapse had an EpCAM-negative/E-cadherin-positive tumor.

The disagreement, observed between the inverse prognostic role of these two adhesion molecules and the strong positive association found by multiple logistic regression (visualized also by MCA) is only apparent. Indeed, the 
positive association between EpCAM and E-cadherin expression can be explained by the notion that most epithelial cell types coexpress E-cadherin and Ep-CAM during some stage of embryogenesis (Litvinov, 1995). In addition, experimental findings have clearly demonstrated that overexpression of EpCAM has no influence on the expression nor the number of the cadherin molecules, but affects only the interaction of cadherins with the cytoskeleton leading to the gradual abrogation of junctional complex. In other words, as the cadherin-mediated cell-cell adhesions diminishes, EpCAM-mediated intercellular connection become predominant whereas cadherins, which have lost their connection to the cytoskeleton, remain present and detectable as complexes with $\beta$-catenin (Litvinov, 1997).

In our knowledge, this is the first time that p53 status, EpCAM and E-cadherin expression are jointly investigated in breast cancer clinical specimens, providing information useful for a better characterization of the group of p53-positive tumors and identification of patients with a high risk of recurrence.

\section{Acknowledgements}

We thank Dr Eros Magri and Dr Anna Cherubino for technical assistance. This work was supported in part by the Fondazione of the Cassa di Risparmio della Provincia di Chieti, the EU NoE Biopattern (FP6-2002-IST-1 no. 508803), the Italian Association for Cancer Research (AIRC, Italy), ABO and ABO Project SpA (Grant VE01D0019).

\section{References}

Alberti, S., Ambrogi, F., Boracchi, P., Fornili, M., Querzoli, P., Pedriali, M., ... Coradini, D. (2012). Cytoplasmic Trop-1/Ep-CAM Overexpression is Associated with a Favorable Outcome in Node-Positive Breast Cancer. Japanese Journal of Clinical Oncology, 42(12), 1128-1137. http://dx.doi.org/10.1093/jjco/hys159

Ambrogi, F., Biganzoli, E., Querzoli, P., Ferretti, S., Boracchi, P., Alberti, S., ... Nenci, I. (2006). Molecular subtyping of breast cancer from traditional tumor marker profiles using parallel clustering methods. Clinical Cancer Research, 12(3 Pt 1), 781-790. http://dx.doi.org/10.1158/1078-0432.CCR-05-0763

Biganzoli, E., Coradini, D., Ambrogi, F., Garibaldi, J. M., Lisboa, P., Soria, D., ... Alberti, S. (2011). P53 Status Identifies Two Subgoups of Triple-negative Breast Cancers with Distinct Biological Features. Japanese Journal of Clinical Oncology, 41(2), 172-179. http://dx.doi.org/10.1093/jjco/hyq227

Chae, B. J., Bae, J. S., Lee, A., Park, W. C., Seo, Y. J., Song, B. J., ... Jung, S. S. (2009). P53 as a specific prognostic factor in triple-negative breast cancer. Japanese Journal of Clinical Oncology, 39(4), 217-224. http://dx.doi.org/10.1093/jjco/hyp007

Cheok, C. F., Verma, C. S., Baselga, J., \& Lane, D. P. (2011). Translating p53 into the clinic. Nature Reviews Clinical Oncology, 8(1), 25-37. http://dx.doi.org/10.1038/nrclinonc.2010.174

Cimino, A., Halushka, M. M., Illei, P., Wu, X., Sukumar, S., \& Argani, P. (2010). Epithelial cell adhesion molecule (EpCAM) is overexpressed in breast cancer metastases. Breast Cancer Research and Treatment, 123(3), 701-708. http://dx.doi.org/10.1007/s10549-009-0671-z

Di Leo, A., Tanner, M., Desmedt, C., Paesmans, M., Cardoso, F., Durbecq, V., ... Isola, J. (2007). P53 gene mutations as a predictive marker in a population of advanced breast cancer patients randomly treated with doxorubicin or docetaxel in the context of a phase III clinical trial. Annals of Oncology, 18, 997-1003. http://dx.doi.org/10.1093/annonc/mdm075

Fine, J. P., \& Gray, R. (1999). A proportional hazard model for the subdistribution of a competing risk. Journal of American Statistics Association, 94, 496-509. http://dx.doi.org/10.1080/01621459.1999.10474144

Gastl, G., Spizzo, G., Obrist, P., Dünser, M., \& Mikuz, G. (2000). Ep-CAM over-expression in breast cancer as a predictor of survival. Lancet, 356(9246), 1981-1982. http://dx.doi.org/10.1016/S0140-6736(00)03312-2

Greenacre, M. J. (2007). Correspondence Analysis in Practice ( ${ }^{\text {nd }}$ Edition), Chapman \& Hall/CRC: London. http://dx.doi.org/10.1201/9781420011234

Guerra, E., Lattanzio, R., La Sorda, R., Dini, F., Piantelli, M., Tiboni, G. M., \& Alberti, S. (2012). mTrop1/EpCAM knockout mice develop congenital tufting enteropathy through disegulation of intestinal E-cadherin/ $\beta$-catenin PLosONE (in press). http://dx.doi.org/10.1371/journal.pone.0049302

Hermiston, M. L., \& Gordon, J. I. (1995). In vivo analysis of cadherin function in the mouse intestinal epithelium: essential roles in adhesion, maintenance of differentiation, and regulation of programmed cell death. Journal of Cell Biology, 129(2), 489-506. http://dx.doi.org/10.1083/jcb.129.2.489

Kimura, H., Kato, H., Faried, A., Sohda, M., Nakajima, M., Fukai, Y., ... Kuwano, H. (2007). Prognostic 
significance of EpCAM expression in human esophageal cancer. International Journal of Oncology, 30(1), 171-179.

Langerod, A., Zhao, H., Borgan, O., Nesland J. M., Bukholm, I. R., Ikdahl, T., ... Jeffrey, S. S. (2007). TP53 mutation status and gene expression profiles are powerful prognostic markers of breast cancer. Breast Cancer Research, 9, R30. http://dx.doi.org/10.1186/bcr1675

Levine, A. J., \& Oren, M. (2009). The first 30 years of p53: growing ever more complex. Nature Reviews Cancer, 9(10), 749-758. http://dx.doi.org/10.1038/nrc2723

Litvinov, S. V., Velders, M. P., Bakker, H. A., Fleuren. G. J., \& Warnaar, S. O. (1994). Ep-CAM: a human epithelial antigen is a homophilic cell-cell adhesion molecule. Journal of Cell Biology, 125(2), 437-446. http://dx.doi.org/10.1083/jcb.125.2.437

Litvinov, S. V. (1995). Ep-CAM: a homophilic cell-cell adhesion molecule with EGF-like domains. Trends in Glycoscience and Glycotechnology, 7(37), 375-384. http://dx.doi.org/10.4052/tigg.7.375

Litvinov, S. V., Balzar, M., Winter, M. J., Bakker, H. A. M., Briaire-de Bruijn, I. H., Prins, F. A., ... Warnaar, S. O. (1997). Epithelial adhesion molecule Ep-CAM modulates cell-cell interactions mediated by classic cadherins. Journal of Cell Biology, 139(5), 1337-1348. http://dx.doi.org/10.1083/jcb.139.5.1337

Marubini, E., \& Valsecchi, M. G. (1995). Analysing Survival Data from Clinical Trials and Observational Studies. John Wiley \& Sons: Chichester.

McShane, L. M., Altman, D. G., Sauerbrei, W., Taube, S. E., Gion, M., \& Clark, G. M. (2005). Reporting recommendations for tumour marker prognostic studies. Journal of Clinical Oncology, 23(36), 9067-9072. http://dx.doi.org/10.1200/JCO.2004.01.0454

Momburg, F., Moldenhauer, G., Hammerling, G. J., \& Mollers, P. (1987). Immunohistochemical study of the expression of a Mr 34,000 human epithelium-specific surface glycoprotein in normal and malignant tissues. Cancer Research, 47(2883), 2883-2891.

Munz, M., Kieu, C., Mack, B., Schmitt, B., Zeidler, R., \& Gires, O. (2004). The carcinoma-associated antigen EpCAM upregulates c-myc and induces cell proliferation. Oncogene, 23(34), 5748-5758. http://dx.doi.org/10.1038/sj.onc.1207610

Olivier, M., Langerod, A., Carrieri, P., Bergh, J., Klaar, S., Eyfjord, J., ... Borresen-Dale, A.-L. (2006). The clinical value of somatic TP53 gene mutations in 1,794 patients with breast cancer. Clinical Cancer Research, 12(4), 1157-1167. http://dx.doi.org/10.1158/1078-0432.CCR-05-1029

Olivier, M., Hollstein, M., \& Hainaut, P. (2010). TP53 mutations in human cancers: origins, consequences, and clinical use. Cold Spring Harbor Perspective in Biology, 2, a001008. http://dx.doi.org/10.1101/cshperspect.a001008

Osta, W. A., Chen, Y., Mikhitarian, K., Mitas, M., Salem, M., Hannun, Y. A., ... Gillanders, W. E. (2004). EpCAM Is Overexpressed in Breast Cancer and Is a Potential Target for Breast Cancer Gene Therapy. Cancer Research, 64(16), 5818-5824. http://dx.doi.org/10.1158/0008-5472.CAN-04-0754

R Development Core Team. (2011). R: A language and environment for statistical computing. R Foundation for Statistical Computing, Vienna, Austria., ISBN 3-900051-07-0, URL http://www.R-project.org/

Riley, T., Sontag, E., Chen, P., \& Levine, A. (2008). Transcriptional control of human p53-regulated genes. Nature Reviews Molecular Cell Biology, 9(5), 402-412. http://dx.doi.org/10.1038/nrm2395

Rosales, C., O'Brien, V. Kornberg, L., \& Juliano, R. (1995). Signal transduction by cell adhesion receptors. Biochemica et Biophysica Acta, 1242(1), 77-98.

Sankpal, N. V., Willman, M. W., Fleming, T. P., Mayfield, J. D., \& Gillanders, W. E. (2009). Transcriptional repression of epithelial cell adhesion molecule contributes to p53 control of breast cancer invasion. Cancer Research, 69(3), 753-757. http://dx.doi.org/10.1158/0008-5472.CAN-08-2708

Schemper, M., \& Smith, T. L. (1996). A note on quantifying follow-up in studies of failure time. Controlled Clinical Trials, 17(4), 343-346. http://dx.doi.org/10.1016/0197-2456(96)00075-X

Schmidt, M., Hasenclever, D., Schaeffer, M., Boehm, D., Cotarelo, C., Steiner, E., ... Schuler, M. (2008). Prognostic Effect of Epithelial Cell Adhesion Molecule Over-expression in Untreated Node-Negative Breast Cancer. Clinical Cancer Research, 14(18), 5849-5855. http://dx.doi.org/10.1158/1078-0432.CCR-08-0669 
Schön, M. P., Schön, M., Mattes, M. J., Stein, R., Weber, L., Alberti, S., \& Klein, C. E. (1993). Biochemical and immunological characterization of the human carcinoma- associated antigen MH 99/KS 1/4. International Journal of Cancer, 55(6), 988-995. http://dx.doi.org/10.1002/ijc.2910550619

Seligson, D. B., Pantuvk, A. J., Liu, Huang Y., Horvath, S., Bui, M. H., Han, K. R., ... Figlin, R. A. (2004). Epithelial cell adhesion molecule (KSA) expression: pathobiology and its role as an independent predictor of survival in renal cell carcinoma. Clinical Cancer Research, 10(8), 2659-2669. http://dx.doi.org/10.1158/1078-0432.CCR-1132-03

Spizzo, G., Went, P., Dirnhofer, S., Obrist, P., Simon, R., Spichtin, H., ... Gastl, G. (2004). High Ep-CAM expression is associated with poor prognosis in node-positive breast cancer. Breast Cancer Research and Treatment, 86(3), 207-213. http://dx.doi.org/10.1023/B:BREA.0000036787.59816.01

Spizzo, G., Fong, D., Wurm, M., Ensinger, C., Obrit, P., Hofer, C., ... Went, P. (2011). EpCAM expression in primary tumour tissues and metastases: an immunohistochemical analysis. Journal of Clinical Pathology, 64(5), 415-420. http://dx.doi.org/10.1136/jcp.2011.090274

Suzuki, K., \& Matsubara, H. (2011). Recent advances in p53 research and cancer treatment. Journal of Biomedicine \& Biotechnology, 2011, 978312. http://dx.doi.org/10.1155/2011/978312

Takeichi, M. (1995). Morphogenic roles of classic cadherins. Current Opinions on Cell Biology, 7(5), 619-627. http://dx.doi.org/10.1016/0955-0674(95)80102-2

Trzpis, M., Popa, E. R., McLaughlin, P. M., van Goor, H., Timmer, A., Bosman, G. W., ... Harmsen, M. C. (2007). Spatial and temporal expression patterns of the epithelial cell adhesion molecule (EpCAM/EGP-2) in developing and adult kidneys. Nephron. Experimental Nephrology, 107(4), e119-e131. http://dx.doi.org/10.1159/000111039

Went, P. T., Lugli, A., Meier, S., Bundi, M., Mirlacher, M., Sauter, G., \& Dirnhofer, S. (2004). Frequent EpCAM protein expression in human carcinomas. Human Pathology, 35(1), 122-128. http://dx.doi.org/10.1016/j.humpath.2003.08.026

Went, P., Dirnhofer, S., Schöpf, D., Moch, H., \& Spizzo, G. (2008). Expression and prognostic significance of EpCAM. Journal of Cancer Molecules, 3, 169-174.

Winter, M. J., Nagelkerken, B., Mertens, A. E., Rees-Bakker, H. A., Briaire-de Bruijn, I. H., \& Litvinov, S. V. (2003). Expression of Ep-CAM shifts the state of cadherin-mediated adhesions from strong to weak. Experimental Cell Research, 285(1), 50-58. http://dx.doi.org/10.1016/S0014-4827(02)00045-9 